\title{
La résolution des traumatismes en complément du traitement standard des abuseurs d'enfants : étude qualitative
}

\author{
Ronald J. Ricci \\ Resources for Human Development, Philadelphia, Pennsylvania \\ Cheryl A. Clayton \\ The Counseling Center, Richlands, Virginia
}

\begin{abstract}
Une analyse documentaire des modèles actuels de traitement des agresseurs d'enfants et des théories contemporaines de l'étiologie donne à penser qu'il existe un écart entre la théorie et la pratique. On reconnaît de plus en plus l'importance d'aborder les questions étiologiques dans le traitement des délinquants sexuels, et pourtant de nombreux programmes sont réticents à aborder les séquelles traumatiques d'abus sexuels infantiles (ASI) chez les délinquants sexuels qui en ont subi. L'ajout du traitement des traumatismes au traitement standard des délinquants sexuels est apparu comme un moyen de combler une partie de cet écart. Dix agresseurs d'enfants ayant rapporté avoir des antécédents d'ASI ont ainsi été traités par EMDR (désensibilisation et retraitement par les mouvements oculaires). Suite à l'ajout de cette composante de résolution des traumatismes, on a constaté des améliorations dans les six sous-échelles de l'Échelle d'évaluation du traitement des délinquants sexuels ainsi qu'une diminution de l'excitation idiosyncrasique déviante, mesurée par le pléthysmographe pénien. La présente étude passe en revue les données qualitatives recueillies au cours du traitement et des entretiens post-traitement.
\end{abstract}

Mots-clés: agresseur sexuel; abuseur d'enfants; EMDR; traitement TCC-PR (prévention des rechutes); traitement qualitatif.

es abus sexuels infantiles (ASI) sont un problème social qui a éveillé beaucoup d'attention à la fois dans le grand public et chez les professionnels au cours des deux dernières décennies. La couverture sensationnelle de cas très médiatisés a permis de faire sortir cette question de l'ombre et de sensibiliser le public. Les effets de l'ASI modifient la vie de la victime, et l'ASI atteint des fréquences inquiétantes.

Une revue par Walker, Carey, Mohr, Stein, and Seedat (2004), portant sur 11 études internationales de
l'ASI, révèle des taux de prévalence de 5,8 à $34 \%$ chez les filles et de 2 à $11 \%$ chez les garçons. Compte tenu de cette fréquence élevée, il est regrettable qu'une étude longitudinale récente (Marques, Wiederanders, Day, Nelson, \& van Ommeren, 2005) indique que l'approche thérapeutique utilisée dans la majorité des programmes de traitement des délinquants sexuels (McGrath, Cummings, \& Burchard, 2003) donne, avec cette population difficile, des résultats décevants.

This article originally appeared as Ricci, R. J., \& Clayton, C. A. (2008). Trauma resolution treatment as an adjunct to standard treatment for child molesters: A qualitative study. Journal of EMDR Practice and Research, 2(1), 41-50. doi:10.1891/19333196.2.1.41. Translated by F. Mousnier-Lompré. 
Hanson et ses collaborateurs (2002) ont effectué une méta-analyse de 42 études mondiales sur le traitement des délinquants sexuels, portant sur 9454 participants. Leurs résultats ont révélé des taux de récidive plus faibles $(12,3 \%)$ chez les délinquants traités que chez les membres du groupe témoin (16,8\%). (Il est à noter que la récidive implique une nouvelle arrestation, mais ne représente sans aucun doute pas tous les nouveaux incidents d'abus sexuel.) Le Projet de traitement et d'évaluation des délinquants sexuels (Sex Offender Treatment and Evaluation Project) est une étude longitudinale globale qui a exploré l'efficacité du traitement intensif TCC-PR (Prévention des Récidives) sur la récidive d'abus sexuels. La conclusion de ces chercheurs, anticipée depuis longtemps, était que leurs constatations "ne confirment pas, de façon générale, l'efficacité du modèle PR" (Marques et coll., 2005, p. 79). Brooks-Gordon, Bilby, and Wells (2006) ont passé systématiquement en revue neuf essais contrôlés randomisés d'interventions TCC auprès de délinquants sexuels, publiées entre 1998 et 2003. Ils ont conclu que le traitement réduisait les taux de nouvelles arrestations à un an, mais qu'il augmentait celles-ci à dix ans. Ces résultats décevants ont abouti à un appel en faveur de traitements plus efficaces pour cette population difficile.

Selon certains théoriciens, des difficultés développementales contribueraient à l'étiologie des voies de délinquance sexuelle (Marshall \& Barbaree, 1990) et perturberaient l'engagement du patient dans le traitement, augmentant ainsi le risque de récidive (Beech \& Ward, 2006). La théorie et la recherche étiologiques suggèrent qu'une voie vers la délinquance chez certains abuseurs est celle d'un trouble du développement sexuel (p. ex. un traumatisme sexuel dans l'enfance;Schwartz, 1994 ; Ward, Hudson, \& Keenan, 1998; Ward \& Siegert, 2002). Van der Kolk (1996) suggère que les effets du traumatisme peuvent devenir le noyau autour duquel sont organisés les comportements et même la personnalité du sujet. Les séquelles d'un traumatisme peuvent comprendre des ruptures d'attachement et de confiance (James, 1989), une rage non traitée (Schwartz, 1994), un retard de développement social et affectif et une sexualité et/ou une excitation anormales (Finkelhor, 1986 ; Money, 1986). Le traumatisme peut aussi désintégrer tout sentiment d'avoir un avenir (Fletcher, 1996 ; Terr, 1991), favorisant ainsi la propension à rechercher une satisfaction immédiate. Ce sont là des traits qui caractérisent de nombreux agresseurs d'enfants.

Le modèle des cheminements de Ward et Siegert (2002), qui combine les éléments les plus solides des théories multifactorielles existantes (p. ex. Finkelhor, 1984 ; Hall \& Hirschman, 1991 ; Marshall \& Barbaree, 1990), est en cohérence à ces idées et a été l'objet de beaucoup d'attention. Le modèle des cheminements attribue les phénomènes cliniques qui sont évidents chez les délinquants sexuels à quatre mécanismes psychologiques interactifs : déficits d'intimité et de compétence sociale, scénarios sexuels déformés, dysrégulation émotionnelle, et croyances allant dans le sens du délit. Ils identifient également une voie étiologique qui intègre ces quatre mécanismes à des scripts sexuels déviants. Ceux-ci reflètent habituellement des antécédents d'abus sexuel ou d'exposition prématurée à la sexualité.

Il subsiste toutefois un écart entre la théorie et la pratique, puisque l'abord de l'ASC chez les délinquants sexuels n'est toujours pas une composante standard de la plupart des programmes de traitement des délinquants sexuels adultes (Becker \& Murphy, 1998 ; Pithers, 1990 ; Salter, 1988). Dans notre propre travail auprès de délinquants sexuels, nous avons observé que certains délinquants ayant un passé de victime d'abus sexuel pendant l'enfance ont des souvenirs déformés de ces agressions ou un engourdissement des émotions qui s’y rapportent. Cela rend difficile, voire impossible, l'accès au matériel concernant leur infraction sexuelle, alors qu'il s'agit d'un élément important du traitement TCC-PR. Par exemple, pour se protéger des souvenirs pénibles de l'ASI, l'abuseur développe des distorsions cognitives qui l'amènent à nier à ses propres yeux (et à l'égard des autres) qu'il ait subi un dommage de l'agression qu'il a connue, ou à croire que c'est lui, l'enfant, qui était responsable de l'abus. Le délinquant généralise ensuite cela à d'autres personnes, y compris à sa ou ses victimes. En d'autres termes, il développe la conviction que si l'acte dont il a été victime ne lui a pas fait de mal, son propre acte ne fait pas de mal à sa ou ses victimes. Chez d'autres, le fait de se croire responsables de ce qu'ils ont subi dans leur enfance les amène, à l'âge adulte, à transférer sur leurs victimes la responsabilité des infractions qu'ils commettent sur elle. L'abuseur construit alors des croyances déformées pour appuyer l'idée que s'il n'a fait aucun mal et qu'il n'est pas responsable de ses actes, il n'est pas nécessaire d'entreprendre un traitement visant à le faire changer.

Nous avons également remarqué que certains des patients de notre programme de traitement des délinquants sexuels ont progressé plus lentement que prévu malgré une motivation apparemment forte. Nous avons soupçonné que, dans certains cas, un trauma 
infantile non résolu les empêchait de s'engager pleinement dans le travail émotionnel indispensable pour explorer et internaliser les concepts du traitement TCC-PR standard. De plus, nous avons observé des situations où les abuseurs construisaient de solides plans de prévention des rechutes, mais se heurtaient à des problèmes persistants de surcharge émotive qui déclenchaient alors leur cycle d'abus. Cette idée est corroborée par les résultats d'une étude menée en 2002 sur les délinquants sexuels juvéniles et les traumatismes (McMackin, Leisen, Cusack, LaFratta, \& Litwin, 2002). Ces croyances et ces comportements distordus peuvent ne pas être délibérés ou conscients, ils sont plutôt la manifestation de la perspective qui accompagne leurs souvenirs des abus subis dans leur enfance (Shapiro, 1995, 2001). Les théories et les croyances implicites qui en résultent et qui se développent à travers ces expériences influencent les perceptions et le comportement ultérieur de ces personnes (Dweck, Chiu, \& Hong, 1995). Dans la littérature sur les délinquances sexuelles, Marshall and Laws (2003) reconnaissent cette notion, avec l'idée que ces distorsions cognitives, courantes chez les délinquants sexuels, peuvent en fait être des théories implicites (Ward, 2000) aussi bien qu'explicites. Par rapport à ces théories, les auteurs proposent que, si on résout l'ASI de l'abuseur, en ajoutant au traitement TCC-PR standard un élément de résolution des traumatismes, on augmente la capacité de l'abuseur à entreprendre une thérapie. On améliore ainsi les résultats du traitement.

Nous avons choisi la méthode de désensibilisation et de retraitement par les mouvements oculaires (EMDR ; Shapiro, 2001) comme moyen de traiter le traumatisme. L'EMDR est un traitement utilisé pour cibler et traiter les souvenirs perturbants, ce qui permet d'éliminer les sensations associées et de faire passer les composantes cognitives et affectives à une forme adaptée. L'EMDR est reconnue dans la revue Cochrane comme l'un des deux traitements efficaces pour les états de stress post-traumatique (ESPT ; Bisson \& Andrew, 2007), et elle est recommandée par l'American Psychiatric Association (2004) comme l'un des meilleurs traitements pour ce trouble. On a également montré que l'EMDR est efficace dans le traitement du trauma (p. ex. Bradley, Greene, Russ, Dutra, \& Westen, 2005; Hertlein \& Ricci, 2004 ; Maxfield \& Hyer, 2002) ; elle a donné de bons résultats auprès d'autres populations présentant des comportements problématiques (Datta \& Wallace, 1994 ; Greenwald, 2002 ; Soberman, Greenwald, \& Rule, 2002). Ricci (2006) a montré que l'EMDR était utile pour le traitement des traumatismes chez un agresseur d'enfants, comme en témoignait l'augmentation de la motivation au traitement et des réactions empathiques chez ce dernier.

Dans l'étude dont il est question dans le présent document, Ricci, Clayton et Shapiro (2006) ont constaté que l'ajout de l'EMDR au traitement TCC-PR standard entraînait une amélioration statistiquement significative avant/après traitement sur les six souséchelles de l'Échelle d'évaluation du traitement des délinquants sexuels (Anderson, Gibeau, \& D’Amora, 1995), qui est conçue pour mesurer les progrès sur des questions spécifiques au traitement par TCC-PR. De plus, les résultats ont montré une réduction significative de l'excitation sexuelle déviante, mesurée par le pléthysmographe pénien, réduction qui n'était pas observée dans un groupe témoin.

L'étude originale (Ricci et coll., 2006) comparait les abuseurs qui avaient reçu un traitement EMDR d'appoint pendant le programme TCC-PR. L'objectif de la présente étude était de faire une analyse qualitative des expériences des participants au cours de cette étude afin d'explorer les processus sous-jacents qui avaient pu contribuer aux résultats.

Nous avons posé l'hypothèse que les distorsions cognitives que l'on retrouve habituellement chez les abuseurs d'enfants étaient en partie causées par les souvenirs des ASI subis par les patients eux-mêmes et qu'elles se manifesteraient au cours du traitement EMDR du traumatisme. Nous avons également émis l'hypothèse que ces distorsions se modifieraient pour prendre une forme adaptée au cours des séances d'EMDR et apparaîtraient donc dans l'analyse de la théorie ancrée.

\section{Méthode}

\section{Participants}

Dix hommes condamnés pour des agressions sexuelles sur des enfants ont été sélectionnés pour suivre un traitement EMDR en complément de leur programme standard de traitement TCC-PR pour délinquants sexuels. Tous étaient en probation et/ou en libération conditionnelle dans la collectivité et sous obligation de soins ; leur participation à cette étude était néanmoins facultative. Les participants provenaient de plusieurs sites d'un programme communautaire TCC-PR dans l'est des États-Unis. Les participants suivaient le traitement TCC-PR depuis en moyenne 28 mois (entre 6 mois et 5 ans 8 mois). Ils étaient âgés de 18 à 52 ans, avec une moyenne d'âge de 37,5 ans. Tous étaient blancs. L'un d'eux a signalé prendre des psychotropes pour traiter un trouble de l'humeur. Tous les patients avaient un emploi : 
l'éventail allait de techniciens et de professionnels qualifiés, ayant des revenus substantiels, à des personnes entrant dans les critères de pauvreté.

Tous les participants avaient signalé des antécédents d'ASI plus tôt dans leur programme de TCC-PR. Tous ne percevaient pas ces ASI comme problématiques ou néfastes pour eux, et ils n'attribuaient pas non plus leur propre délinquance sexuelle aux ASI qu'ils avaient subis. On constatait cependant que tous avaient du mal à s'engager pleinement dans leur thérapie TCC-PR, comme l'indiquait la présence d'au moins un des éléments suivants : (a) une incapacité à discuter des particularités de leur délinquance sexuelle sans se retrouver bloqués dans la thérapie ; (b) l'inquiétude du clinicien que les progrès du traitement ne soient plus cognitifs qu'émotionnels, c'est-à-dire qu'il n'y avait pas d'internalisation du préjudice causé à la victime ni de conscience du risque de récidive ; ou (c) l'inquiétude du clinicien parce que des déclencheurs émotionnels activaient régulièrement les comportements déviants et aggravaient donc les risques de récidive. Chaque participant a signé un formulaire de consentement éclairé approuvé par un comité d'examen institutionnel. Le consentement décrivait l'objectif du projet, les procédures, les risques, les avantages, la confidentialité et la liberté de se retirer à tout moment sans conséquence. Le comité de recherche du département d'État chargé de la correction avait également donné son feu vert au projet.

\section{L'EMDR}

L'EMDR a d'abord été développée comme traitement pour les victimes de traumatisme émotionnel (Shapiro, 1989, 1995, 2001). Il s'agit d'un traitement en huit phases qui comprend la collecte des antécédents du patient (phase 1), la préparation du patient à l'intensité de la thérapie du trauma (phase 2), l'évaluation du niveau de détresse et des composantes du souvenir traumatique cible (phase 3) et l'utilisation d'une forme de stimulation bilatérale pour désensibiliser et traiter les souvenirs traumatiques (phases 4-6). Au cours de cette phase de traitement, des procédures normalisées guident l'attention du patient, auquel le thérapeute demande d'être attentif à divers aspects du réseau mnésique. Dans un premier temps, le patient se concentre sur le souvenir perturbateur, avec les cognitions et les émotions qui l'accompagnent. Le thérapeute fournit une stimulation bilatérale sous la forme d'un suivi visuel, d'un stimulus auditif ou d'une stimulation tactile. Les progrès du traitement sont évalués à l'aide de l'échelle des unités subjectives de perturbation (SUDS;
Wolpe, 1982) et de l'échelle de validité de la cognition (Shapiro, 1989). Les phases finales du traitement permettent de clôturer la séance et de réévaluer l'état $\mathrm{du}$ souvenir traumatique. Les cibles de traitement pour un traitement complet comprennent les souvenirs qui ont été à la base des dysfonctionnements, les déclencheurs des perturbations actuelles et les "modèles" pour un fonctionnement futur adapté.

\section{Outils et Mesures}

Les SUDS. Il s'agit d'une mesure de processus utilisée en EMDR dans laquelle on demande aux participants de se rappeler la mémoire pertinente au traumatisme et la cognition négative associée et d'évaluer le niveau d'anxiété qui l'accompagne en utilisant une échelle de 11 points, dans laquelle 0 représente une intensité neutre et 10 correspond à la perturbation la plus grande possible (Shapiro, 1989 ; Wolpe, 1982).

L'Échelle d'évaluation du traitement des délinquants sexuels. L'Échelle d'évaluation du traitement des délinquants sexuels (EETDS) est conçue pour évaluer à la fois le processus et les mesures de résultats du traitement cognitivo-comportemental des délinquants sexuels. Dans l'EETDS, six sous-échelles sont propres au traitement cognitivo-comportemental des délinquants sexuels. "Ce sont : l'échelle de prise de conscience (compréhension de l'infraction commise), des pensées déviantes (impulsions liées à l'infraction), de la conscience des risques situationnels (mettant en cause la capacité de maîtrise de soi du sujet), de la motivation (pour le changement personnel grâce au traitement), de l'empathie envers la victime (impact émotionnel des délits sexuels), et l'échelle de la divulgation des infractions" (Anderson et coll., 1995, pp. 223 à 234). Une échelle de Likert de 1 à 6 est utilisée pour évaluer les dimensions comportementales, les chiffres les plus élevés représentant une amélioration. Les données initiales de fiabilité de l'EETDS, fondées sur un échantillon de 122 délinquants sexuels, ont montré que la cohérence interne, l'accord entre les évaluateurs et la fiabilité test-retest étaient tous élevés (Anderson et coll., 1995).

\section{La théorie ancrée}

Nous avons procédé à une analyse de théorie ancrée fondée sur une étude de cas collective et transversale. Les transcriptions des séances de thérapie et d'interview, ainsi que les auto-déclarations des clients et les observations cliniques consignées dans les carnets de recherche, ont été évaluées à l'aide d'un processus de théorie ancrée (Glaser \& Strauss, 1967). 
Les données qualitatives ont été analysées en vue de dégager des thèmes de fond émergents qui ont été développés par codification ouverte et axiale. La méthodologie de la théorie ancrée consiste à collecter et à analyser systématiquement des données à travers les processus de comparaison constante, d'encodage et de codification thématique ou axiale. Elle comprend des techniques de conceptualisation de la façon dont sont reliés les codes de fond, suggérant par là une hypothèse ou une proposition qui se dégage des données (Creswell, 1998; Strauss \& Corbin, 1994). La comparaison constante est le processus qui consiste à comparer les informations provenant du recueil des données aux catégories émergentes. Ces catégories émergent du processus de codage ouvert et sont ensuite rassemblées par codage thématique ou axial, comme décrit précédemment (Creswell, 1998). "Le principe de base de cette méthode est que l'analyse est inductive plutôt que déductive, et que la théorie doit émerger des données" (Strauss \& Corbin, 1990, p. 23).

\section{Procédure}

Le traitement EMDR a été effectué par un clinicien du niveau du doctorat, qui a le niveau II de l'Institut d'EMDR, et qui a 7 ans de pratique de l'EMDR. Par ailleurs, un clinicien de niveau maitrise, ayant également le niveau II de l'Institut d'EMDR, a passé en revue les notes de processus et les bandes vidéo des séances EMDR pour vérifier la conformité du protocole EMDR. Tous les traitements de TCC-PR ont été menés par une équipe clinique à plein temps, agréée et accréditée par l'État, et qui était au courant de notre étude. Le clinicien principal de chaque participant a rempli l'EETDS avant-EMDR. En moyenne, on a mené six séances EMDR avec les dix patients du groupe de traitement avec adjonction d'EMDR, en recourant aux protocoles standardisés (Shapiro, 2001).

Les cibles EMDR ont été choisies au moyen d'entretiens guidés par leurs révélations antérieures d'ASI. Dans les cas où les patients percevaient leur ASI comme traumatisante, ils ont été guidés dans le choix du souvenir-cible le plus perturbant d'agression sexuelle dont ils pouvaient se souvenir. $S$ ' ils ne percevaient pas l'ASI comme traumatisante, un souvenir cible a été négocié entre le patient et le thérapeute. Le traitement demeurait axé sur une ou plusieurs cibles spécifiquement liées à l'ASI qui, pensait-on, interféraient dans l'engagement du patient à l'égard du traitement TCC-PR. Un écart notable par rapport au protocole EMDR standard (Shapiro, 2001) est qu'on considérait la phase de désensibilisation EMDR comme terminée lorsque les rapports des participants sur le SUD 0-10, quant à leur perturbation, étaient aussi bas qu'ils le pensaient possible. Dans deux cas, il est resté un certain niveau de perturbation. Dans ces cas, il semble que la prise de conscience des participants de leur propre victimisation infantile, ainsi que celle du mal qu'ils avaient fait à leurs victimes, empêchait leur SUD d'arriver à une absence de perturbation. Le jugement clinique dans ces cas a été que le fait de laisser subsister un certain degré de culpabilité ou de perturbation au sujet de leurs propres infractions pouvait servir à prévenir la rechute.

Une fois le traitement EMDR terminé, le principal clinicien TCC-PR complétait l'EETDS à des fins de comparaison. Les participants ont été interrogés au sujet de leurs expériences avec l'EMDR ; des chercheurs indépendants qui n'avaient pas fourni le traitement EMDR ni le traitement TCC-PR ont mené les entretiens. Afin de minimiser les biais, les intervieweurs étaient des cliniciens en santé mentale sans formation en EMDR et qui étaient inconnus des participants. Ils utilisaient les questions de l'interview comme guide pour poser aux participants des questions ouvertes sur leur expérience de l'EMDR, le traitement, les bénéfices et les inconvénients de l'EMDR. Nous avions conçu les questions en gardant à l'esprit le point suivant : il fallait que les thèmes émergents soient ceux qui étaient significatifs pour les participants (voir Annexe).

\section{Analyse des données}

Les données ont été analysées à l'aide d'une démarche de théorie ancrée. Elles comprenaient les transcriptions intégrales des séances de traitement et des séances d'interview, ainsi que les auto-déclarations des patients et les observations cliniques consignées dans des carnets de recherche. Une triangulation des sources (Patton, 2002) a été utilisée pour vérifier la cohérence des données et des résultats. Les données qualitatives ont été analysées en vue de faire apparaître des thèmes de fond développés par codage ouvert et axial, conformément à la méthodologie de la théorie ancrée de Glaser et Strauss (1967). Les données ont été analysées au fur et à mesure de leur collecte, puis comparées aux données nouvellement recueillies. Une comparaison ligne par ligne, paragraphe par paragraphe et document par document a été effectuée afin d'identifier et de nommer les concepts qui décrivaient ou expliquaient les phénomènes observés (Strauss $\&$ Corbin, 1994). Les données ont été examinées afin de déceler la présence de patterns émergents recoupant la majeure partie des données (Taylor \& Bogdan, 1984). Les concepts ont été comparés les uns aux autres, et 
ceux qui se rapportaient aux mêmes phénomènes ont été regroupés en catégories en utilisant des thèmes transversaux. Ces thèmes ont été examinés par rapport aux propriétés, au contexte, à l'interaction et aux conséquences des interactions des phénomènes à l'intérieur de chaque thème (Glaser $\&$ Strauss, 1967). Des vérifications des membres ont été menées auprès de plusieurs participants afin de vérifier leurs commentaires et leurs expériences, tels qu'on pouvait se les représenter à partir des thèmes et des conceptualisations qui en découlaient.

\section{Résultats}

\section{Suds}

Les notes de SUD des participants pour le pire souvenir de ce qu'ils avaient subi en tant que victimes, sont descendues d'une moyenne pré-test de 6,9 à une moyenne post-test de 1,9 , une différence statistiquement significative $(t[9]=4,19, p=0,002)$. Cependant, les notes de pré-test de certains participants étaient trompeuses parce qu'ils ont commencé la thérapie en niant absolument que ce qu'ils avaient subi en tant que victimes avait pu leur nuire. Au cours du traitement EMDR, lorsqu'ils se sont rendu compte à quel point l'événement leur avait causé du tort, leurs niveaux de SUD ont augmenté : une fois le déni traité, le niveau moyen de perturbation était de 9,2, ce qui était statistiquement supérieur à la mesure post-test ( $\mathrm{t}$ [9] = $11,69, \mathrm{p}=<.001)$. Bref, il est ressorti clairement de cette mesure du processus de l'EMDR que la procédure était efficace pour réduire les perturbations liées aux souvenirs ciblés.

\section{Analyse Qualitative}

Sept thèmes et deux sous-thèmes de poids et d'importance à peu près égaux ont émergé de l'analyse des données.

Reconnaissance des éléments ayant contribué aux croyances déformées $d u$ patient. Un objectif important du traitement TCC-PR est de réduire le nombre de croyances favorables aux délits sexuels qu'entretiennent les abuseurs à l'égard de leur propre comportement et à l'égard de leurs victimes. Un thème lié à cette question est la capacité des clients de commencer à reconnaître le développement ou l'origine des distorsions cognitives liées à leur comportement délinquant. Voici quelques exemples de la façon dont certains patients ont découvert qu'ils pensaient de façon déformée, ce qu'ils ont relié aux abus qu'ils avaient eux-mêmes subis:
Distorsions cognitives. “Je crois que ce qu'il m'a fait, ça m'a donné l'idée que c'est normal d'avoir des relations sexuelles avec des gamins tant qu'on ne les force pas. Tant qu'ils disent "d'accord."

"Je me souviens que c'est là que j'ai commencé à penser qu'il n'y avait rien de mal à utiliser les gens, tant que ça te fait du bien." "Ça ne faisait pas mal. Il ne me faisait pas mal. Parfois même ça me faisait du bien. J'y pensais beaucoup, après. Je me masturbais en y pensant. Je ne peux pas expliquer pourquoi. Je me suis dit que [ma victime] se sentait comme ça. Qu'il aimait ça, comme moi avant."

"De savoir que j'étais moi-même une victime dans le passé, ça m’a aidé. Je les regarde maintenant, et je me dis : "Ho ! c'est juste un enfant." "Je vois maintenant où [mes victimes] ne voulaient pas vraiment que $\mathrm{j}$ 'aille. Vous voyez, sexuellement ou un truc comme ça. C'était juste qu'ils voulaient qu'on s'occupe d'eux, comme moi avec [son agresseur]."

"Alors qu'avant, je m'étais convaincu que mes victimes voulaient vraiment ce que je leur faisais. Et, euh, je pense que ça m'a vraiment aidé à voir les dégâts que j'ai faits."

"Je vois maintenant que c'était qu'une enfant. Un bébé, vraiment. Et je n'étais qu'un petit enfant moi aussi."

La responsabilité. Également évident : le thème, apparenté au précédent, de la vision déformée qu’ils avaient de leur responsabilité à l'égard de leurs actes délinquants:

"Je lui en voulais pour ce que je faisais, moi. Pour ce que je faisais subir à mes victimes. Je blâmais mon grand-père. Mais après la troisième séance (d'EMDR), j'ai vu que ce n'était pas sa faute. Pas plus que ce n'était la faute de ma victime."

"Je voulais... me prendre pour une victime d'abus et rejeter la responsabilité de ce que j'avais fait, mon [délit], sur tout ça."

Participation accrue à la thérapie de groupe. Les participants ont également décrit les raisons pour lesquelles ils pensaient être d'accord pour être plus actifs dans leurs séances de groupe TCC-PR:

"Je n'ai plus peur de poser des questions [dans le groupe]."

"Ça a changé ma façon de me voir, comme si j'avais relevé la tête et que je m’étais dit que j'étais quelqu'un de bien et que j'avais quelque 
chose à offrir au groupe, et aux autres en général."

Un thème en rapport avec celui-ci est celui d'une impression de concentration plus forte sur les tâches du groupe TCC-PR :

"Je crois que je suis davantage présent, ici, quand je travaille sur mon schéma d'abus sexuel. Je suis plus en contact avec ce qui se passait vraiment avec mes victimes, avec la façon dont je m'y prenais pour les caresser et tout... Ça m'a aidé à voir les deux côtés de tout ça. Avant, j’avais du mal à expliquer mon comportement [d'abus]."

"Il y a deux semaines, j’ai fait ma présentation (sur l'impact sur les victimes), et j'étais, enfin..., ça m’a aidé. J’ai pu en parler, et ça allait."

Augmentation de l'empathie. Un autre objectif du traitement TCC-PR est le développement de l'empathie. Ce thème d'une plus grande empathie était également très répandu dans les données recueillies:

"Ça m’a aidé à mieux me comprendre, moi et les autres."

"Pour ce qui est de faire l'amour avec [ma petite amie], je n'ai jamais été brutal, hein, mais je crois que maintenant je la comprends mieux."

"Alors, comme je dis, si tu ne te comprends pas toi-même, tu ne comprendras pas les autres."

"Ça m'a ouvert les yeux sur ce qu' ils doivent vraiment ressentir, et sur le chagrin et la douleur que je leur ai causés." "Je peux ressentir la douleur de ce que j'ai subi moi-même, et aussi celle de ma victime."

Clarification de la pensée. La métaphore suivante représente un autre thème émergent, à savoir que le traitement EMDR a aidé de façon générale à clarifier la pensée du patient:

"C’était comme si mon esprit était une assiette de spaghetti : quand je le regardais, tout était confus, entortillé. Maintenant, mon esprit, c'est comme s'il y avait de la viande ici, des pommes de terre là, et un légume encore là. C'est comme ça maintenant."

La prise de conscience en tant qu'outil de gestion de soi. Les participants décrivaient également une réduction ou une meilleure gestion des pensées déviantes. Les données suggèrent que les participants mentionnaient une conscience élevée comme un moyen de mieux gérer leurs pensées déviantes:
"Oui, avant, je voyais des choses qui m'excitaient... mais maintenant que j'ai pris conscience de ma propre enfance, je peux essayer de garder un œil sur ça. Et si je tombe sur ça, comme je l'ai dit, je peux m'arrêter maintenant. Je me dis : "Tu n'as pas besoin d'y aller."

"J'ai remarqué qu'avant [certaines choses] m'excitaient un peu. Regarder un film comme celui avec Jodie Foster [Les Accusés] quand elle est victime du... du viol collectif. Et là, $j$ 'avais des idées déviantes à son égard pour ce crime. Et je n'ai pas eu ça avec celui-ci. Ni avec quelques autres films qui sont passés à la télé depuis qu'on fait [de l'EMDR]."

L'estime de soi. L'amélioration de l'estime de soi est également considérée comme un élément important du traitement des délinquants sexuels. Ce thème est également apparu dans les données:

"Le traitement en valait la peine. Je ne sais pas comment $\mathrm{j}$ 'aurais fait pour arriver à me sentir bien dans ma peau. Grâce au traitement, je me suis senti en accord avec moi-même."

"Ça m’a permis de mieux comprendre que c'était comme ça : je ne peux pas m’empêcher d'être comme je suis [homosexuel]. Je n'y peux rien."

Reconnaissance et gestion des émotions. "Et $\mathrm{j}$ 'ai supprimé plein de choses et ça, en fin de compte, ça m’a aidé à arriver là où j'en suis [rire], au pire de ce que je peux être. J'étais incapable de pleurer, je repoussais ça à fond, et quand j'y pensais, je bloquais ça, je le bloquais, je le piétinais, pendant toutes ces années, jusqu'à maintenant." "Dans ma tête, c'était la catastrophe. Je crois que le traitement m'a aidé. Je pense que je peux m'en servir pour prendre le temps de m'asseoir et de réfléchir, au déjeuner ou n'importe quand: m'asseoir, fermer les yeux et me concentrer sur ce qui m'embête vraiment, et puis résoudre la question."

\section{Discussion}

Une limite évidente de cette étude est que le petit nombre de participants a été choisi dans une région concentrée des États-Unis et représente un groupe atypiquement homogène. De plus, si les participants avaient été sélectionnés dans plusieurs centres de traitement, ceux-ci partageaient tous le même programme de traitement et les mêmes références cliniques. Par conséquent, on ne sait pas si les résultats seraient généralisables 
à d'autres programmes, utilisant d'autres techniques, avec d'autres cibles de traitement. Enfin, s'ils n'étaient pas au courant des détails, les évaluateurs cliniques de l'EETDS savaient que les participants suivaient un traitement supplémentaire.

Parmi les points forts de l'étude, en revanche, on notera le fait que les entretiens ont été menés par des cliniciens de niveau maîtrise, qui n'étaient pas au courant des pathologies des participants et qui ne connaissaient ni l'EMDR, ni le but de l'étude. Enfin, malgré des hypothèses peu précises, le guide d'entretien visait à permettre l'émergence de thèmes et de sousthèmes à partir des données.

Il semble que, si on n'aborde pas les traumatismes sexuels personnels du délinquant, l'efficacité du modèle TCC-PR en est réduite. Il est possible que les souvenirs traumatiques non résolus empêchent d'atteindre certains objectifs importants de la TCC-PR.

Rappelons que les objectifs du traitement TCC-PR comprennent la réduction du déni, l'identification de facteurs de risque internes et externes, la formation en compétences sociales, la formation en affirmation de soi, la résolution de problèmes, le reconditionnement de préférences sexuelles déviantes, la restructuration des distorsions cognitives et le développement de l'empathie envers les victimes (Becker \& Murphy, 1998 ; Pithers, 1990 ; Salter, 1988). Ces objectifs principaux sont bien représentés par les thèmes et/ou les sous-thèmes qui sont ressortis de l'analyse des données. La restructuration des croyances favorables au délit est l'une des principales cibles de plus de $90 \%$ des programmes de traitement des délinquants sexuels aux États-Unis (McGrath et coll., 2003). Les données montrent que plus les délinquants participant à cette étude comprenaient grâce à l'EMDR la dynamique de leur ASI, plus ils étaient en mesure de reconnaitre les opinions déformées qu'ils avaient pu avoir de leur délinquance sexuelle. Cette clarification de la responsabilité du délinquant semble avoir ouvert la voie à un plus grand sentiment de responsabilité personnelle. Les résultats montrant une réponse empathique accrue après le traitement EMDR sont conformes à ceux de Datta et Wallace (1994). Bien qu'on ne lui reconnaisse pas un lien étroit avec la récidive (Hanson \& Bussiere, 1998), les participants ont indiqué que leur meilleure capacité d'empathie était un facteur d'amélioration de leurs relations interpersonnelles et d'une plus grande conscience des torts que leurs agressions avaient causés.

Les données suggèrent également une volonté et/ou une capacité accrue des participants à s'engager dans le traitement TCC-PR. Encore une fois, bien que la motivation à l'égard d'un traitement ne soit pas forcément étroitement corrélée aux résultats, l'abandon du traitement a été associé à une augmentation de la récidive (Hanson \& Bussiere, 1998). La participation des patients est considérée comme essentielle par la plupart des théories du changement. Par exemple, la participation et la motivation du patient sont les principales composantes du modèle trans-théorique de changement de Prochaska, Norcross et DiClemente (1994). L'accord du patient et son engagement dans le processus thérapeutique sont fondamentaux dans les procédures de la phase initiale de la thérapie (Brock $\&$ Barnard, 1988). Warren et Green (1995) intègrent des techniques de développement de la motivation dans leur Southwest Sexual Compulsivity Program pour les délinquants sexuels et les obsédés sexuels. Pour eux, le délinquant n'aura de motivation à changer que s'il perçoit des bénéfices au moins équivalents à la difficulté que représente le fait de développer et de maintenir durablement un nouveau mode de vie.

Autre résultat : une meilleure estime de soi. Cela pourrait être lié aux premières étapes du modèle TCC-PR, fréquemment utilisé, de l'infraction sexuelle, selon lequel les émotions mal ou insuffisamment régulées peuvent déclencher le cycle délictueux (Becker \& Murphy, 1998; Ward \& Hudson, 2000).

Les thèmes et les sous-thèmes qui se dégagent des données de l'étude ainsi que des résultats de l’EETDS tendent à appuyer l'idée que le traitement des délinquants sexuels par TCC-PR peut être amélioré par l'ajout d'un volet de résolution des traumatismes, pour ceux d'entre eux qui se souviennent d'avoir été agressés sexuellement dans leur enfance. Les données indiquent que des bénéfices en découlent, que le patient perçoive ou non ses abus d'enfance comme un traumatisme.

Comme l'indique le Tableau 1, les six sous-échelles de l'EETDS conçues pour mesurer les problèmes propres au traitement cognitivo-comportemental des délinquants sexuels ont connu, dans une étude antérieure, une amélioration statistiquement significative avec l'introduction de l'EMDR (Ricci et coll., 2006). Ces résultats concordent avec l'hypothèse concernant les effets de l'ajout d'une composante traumatologique au traitement TCC-PR standard. Les mesures de concept de l'EETDS correspondent en partie aux thèmes et sous-thèmes qui ressortent de l'analyse qualitative de la présente étude. Par exemple, les thèmes de la "reconnaissance par les contributeurs des croyances déformées" et de la "clarification des pensées" sont comparables à la mesure EETDS de la "compréhension". "Meilleure participation à la thérapie de groupe" correspond étroitement 
TABLEAU 1. Données des sous-échelles EETDS pour le groupe de thérapie EMDR $(\mathrm{N}=10)$

\begin{tabular}{lllcc}
\hline Sous-échelles & Pré-EMDR & Post-EMDR & Différence & Valeur $t$ \\
\hline $\begin{array}{l}\text { Prise de con- } \\
\text { science }\end{array}$ & 24 & 37 & 13 & $-4,333^{\star}$ \\
Pensées d'ordre sexuel & 23 & 34 & 11 & $73,498^{\star}$ \\
Conscience du risque & 20 & 32 & 12 & $76,000^{\star}$ \\
Motivation pour le traitement & 30 & 47 & 17 & $73,431^{\star}$ \\
Empathie & 16 & 40,5 & 24,5 & $74,930^{\star}$ \\
Divulgation & 32 & 50 & 18 & $73,250^{\star}$ \\
\hline
\end{tabular}

${ }^{\star} p<.01$

à la catégorie de "motivation pour le traitement". "L'augmentation de la conscience en tant qu'outil d'autogestion" représente le concept EETDS de "conscience du risque", tandis que "empathie accrue" et "empathie" sont évidemment similaires. Cela confirme l'importance des concepts représentés dans l’EETDS.

\section{Conclusion}

Une analyse des multiples points de données recueillis pour cette étude a révélé et appuyé des thèmes suggérant que l'application de l'EMDR avec les agresseurs d'enfants a amélioré le traitement standard spécifique des infractions sexuelles et fait avancer les objectifs de traitement. Ces résultats encourageants font écho aux résultats préliminaires d'autres études qui ont utilisé l'EMDR avec des délinquants sexuels (p. ex. Datta $\&$ Wallace, 1994 ; Finlay, 2002 ; Ricci, 2006 ; Ricci et coll., 2006), ce qui justifie des recherches supplémentaires sur ce modèle de traitement amélioré. Des résultats semblables, obtenus dans le cadre de futures études contrôlées, menées auprès d'un plus grand nombre de participants provenant d'un pool de candidats plus hétérogènes, permettraient de généraliser davantage nos résultats et d'enrichir considérablement la documentation disponible. Une méthode mixte (c.-à-d. quantitative et qualitative) est utile pour examiner certains des concepts (p. ex. la motivation pour le traitement et l'empathie), qui sont difficiles à mesurer. À l'avenir, tous les examinateurs, y compris ceux qui complètent l'EETDS (ou des tests similaires), doivent être aveugles aux conditions des études où ils interviennent. Le recours à un modèle étiologique, comme le modèle des cheminements de Ward et Siegert (2002), pourrait être utile pour étudier les facteurs pouvant contribuer à l'entrée dans la délinquance, comme les scénarios de déviance sexuelle ou les troubles de l'empathie, qui sont liés aux ASI. Si ces idées se confirment dans les études futures, il semblerait bénéfique d'utiliser l'EMDR dès le début d'un programme de traitement TCC-PR. Cela permettrait de mieux préparer les patients au traitement tout en abordant les mécanismes étiologiques de leur délinquance. Ces notions sont cohérentes avec les prédictions du modèle de traitement adaptatif de l'information de Shapiro, qui postule que le traitement des souvenirs perturbants produit une amélioration de la pathologie et des changements dans la perception de soi et des autres. Enfin, la récidive sexuelle demeure sans aucun doute la mesure déterminante des résultats du traitement des délinquants sexuels. Une étude longitudinale contrôlée, faisant un suivi des récidives d'abus sexuels sur enfant, serait donc justifiée pour explorer l'impact de l'intégration de la résolution des traumatismes sur le traitement standard des délinquants sexuels.

\section{Bibliographie}

American Psychiatric Association. (2004). Practice guideline for the treatment of patients with acute stress disorder and post-traumatic stress disorder. Arlington, VA: Author.

Anderson, R., Gibeau, D., \& D’Amora, D. (1995). The sex offender treatment rating scale: Initial reliability data. Sexual Abuse: A Journal of Research and Treatment, 7, 221277. https: / / doi.org/10.1177/107906329500700305

Becker, J. V., \& Murphy, W. D. (1998). What we know and do not know about assessing and treating sex offenders. Psychology, Public Policy and Law, 4, 116-137. https: / / doi.org/10.1037/1076-8971.4.1-2.116

Beech, A., \& Ward, T. (2006). Risk assessment in the 21st century: Towards an integrative model of risk. The Forum: The Association for the Treatment of Sexual Abusers, 18(3), 1-13.

Bisson, J., \& Andrew, M. (2007). Psychological treatment of post-traumatic stress disorder (PTSD). Cochrane Database of Systematic Reviews, 3, CD003388. https: / / doi.org/10.1002/14651858.CD003388.pub3 
Bradley, R., Greene, J., Russ, E., Dutra, L., \& Westen, D. (2005). A multidimensional meta-analysis of psychotherapy for PTSD. American Journal of Psychiatry, 162, 214-227. https://doi.org/10.1176/appi.ajp. 162.2.214

Brock, G. W., \& Barnard, C. P. (1988). Procedures in marriage and family therapy. Needham Heights, MA: Allyn and Bacon.

Brooks-Gordon, B., Bilby, C., \& Wells, H. (2006). A systematic review of psychological interventions for sexual offenders I: Randomised control trials. Journal of Forensic Psychiatry and Psychology, 17, 442-466. https: / / doi.org/10.1080/14789940600819341

Creswell, J. W. (1998). Qualitative inquiry and research design: Choosing among five traditions. Thousand Oaks, CA: Sage.

Datta, P. C., \& Wallace, J. (1994, May). Treatment of sexual traumas of sex offenders using eye movement desensitization and reprocessing. Paper presented at the 11th annual Symposium in Forensic Psychology. SanFrancisco, CA.

Dweck, C. S., Chiu, C., \& Hong, Y. (1995). Implicit theories and their role in judgments and reactions: A world from two perspectives. Psychological Inquiry, 6, 267-285. https: / / doi.org/10.1207/s15327965pli0604_1

Finkelhor, D. (1984). Child sexual abuse: New theory and research. New York, NY: Free Press.

Finkelhor, D. (1986). A sourcebook on child sexual abuse. Beverly Hills, CA: Sage.

Finlay, P. (2002). Eye movement desensitization and reprocessing (EMDR) in the treatment of sex offenders. Dissertation Abstracts International, 63(10). (UMI No. 3068413

Fletcher, K. E. (1996). Childhood posttraumatic stress disorder. In E. Mash \& R. Barkley (Eds.), Child psychopathology (pp. 242-276). New York, NY: Guilford Press.

Glaser, B., \& Strauss, A. (1967). The discovery of grounded theory: Strategies for qualitative research. Chicago: Aldine.

Greenwald, R. (2002). Motivation-adaptive skillstrauma resolution (MASTR) therapy for adolescents with conduct problems: An open trial. Journal of Aggression, Maltreatment, and Trauma, 6, 237-261. https:// doi.org/10.1300/J146v06n01_12

Hall, G. C. N., \& Hirschman, R. (1991). Towards a theory of sexual aggression: A quadripartite model. Journal of Consulting and Clinical Psychology, 59, 662-669. https: / / doi.org/10.1037/0022-006X.59.5.662

Hanson, R. K., \& Bussiere, M. T. (1998). Predicting relapse: A meta-analysis of sexual offender recidivism studies. Journal of Consulting and Clinical Psychology, 66, 348-362. https: / / doi.org/10.1037/0022-006X.66.2.348

Hanson, R. K., Gordon, A., Harris, A. J. R., Marques, J. K., Murphy, W., \& Quinsey, V. L. (2002). First report of the collaborative outcome data project on the effectiveness of psychological treatment for sexual offenders. Sexual Abuse: A Journal of Research and Treatment, 14, 169-194. https: / / doi.org/10.1177/107906320201400207
Hertlein, K. M., \& Ricci, R. J. (2004). A systematic research synthesis of EMDR studies: Implementation of the platinum standard. Trauma, Violence and Abuse: A Review Journal, 5, 285-300. https://doi. org / $10.1177 / 1524838004264340$

James, B. (1989). Treating traumatized children: New insights and creative interventions. Lexington, MA: Lexington Books.

Marques, J. K., Wiederanders, M., Day, D. M., Nelson, C., \& van Ommeren, A. (2005). Effects of a relapse prevention program on sexual recidivism: Final results from California's sex offender treatment and evaluation project (SOTEP). Sexual Abuse: A Journal of Research and Treatment, 17, 79-107. https:// doi.org/10.1177/107906320501700108

Marshall, W. L., \& Barbaree, H. E. (1990). Outcome of comprehensive cognitive behavioral treatment programs. In W. L. Marshall, D. R. Laws, \& H. E. Barbaree (Eds.), The handbook of sexual assault: Issues, theories and treatment of the offender (pp. 363-385). New York, NY: Plenum Press.

Marshall, W. L., \& Laws, D. R. (2003). A brief history of behavioral and cognitive behavioral approaches to sexual offender: Part 2. The modern era. Sexual Abuse: A Journal of Research and Treatment, 15, 93-120. https: / / doi.org/10.1177/1079063n.d.1500202

Maxfield, L., \& Hyer, L. (2002). The relationship between efficacy and methodology in studies investigating EMDR treatment of PTSD. Journal of Clinical Psychology, 58, 23-41. https: / / doi.org/10.1002/jclp.1127

McGrath, R. J., Cummings, G., \& Burchard, B. (2003). Current practices and trends in sexual abuse management. The Safer Society 2002 national survey. Brandon, VT: Safer Society Foundation.

McMackin, R. A., Leisen, M. B., Cusack, J. F., LaFratta, J., \& Litwin, P. (2002). The relationship between trauma exposure to sex offending behavior among male juvenile offenders. Journal of Child Sexual Abuse, 11, 2540. https: / / doi.org/10.1300/J070v11n02_02

Money, J. (1986). Lovemaps: Clinical concepts of sexual/erotic health and pathology, paraphilia, and gender transposition in childhood, adolescence, and maturity. New York, NY: Irvington.

Patton, M. Q. (2002). Qualitative research and evaluation methods (3rd ed.). Thousand Oaks, CA: Sage.

Pithers, W. D. (1990). Relapse prevention with sexual aggressors: A method for maintaining therapeutic change and enhancing external supervision. In W. L. Marshall, D. R. Laws, \& H. E. Barbaree (Eds.), The handbook of sexual assault: Issues, theories and treatment of the offender (pp. 363-385). New York, NY: Plenum Press.

Prochaska, J., Norcross, J. C., \& DiClemente, C. C. (1994). Changing for good. New York, NY: Avon Books.

Ricci, R. J. (2006). Trauma resolution using eye movement desensitization and reprocessing with an incestuous sex offender: An instrumental case study. 
Clinical Case Studies, 5, 248-265. https://doi.org/ $10.1177 / 1534650104265276$

Ricci, R. J., Clayton, C. A., \& Shapiro, F. (2006). Some effects of EMDR on previously abused child molesters: Theoretical reviews and preliminary findings. Journal of Forensic Psychiatry and Psychology, 17, 538-562. https: / / doi.org/10.1080/14789940601070431

Salter, A. C. (1988). Treating child sex offenders and victims: A practical guide. Beverly Hills, CA: Sage.

Schwartz, M. F. (1994). The masters and Johnson treatment program for sex offenders: Intimacy, empathy and trauma resolution. Sexual Addiction and Compulsivity, 1, 261-277. https://doi.org/10.1080/ 10720169408400047

Shapiro, F. (1989). Efficacy of the eye movement desensitization procedure in the treatment of traumatic memories. Journal of Traumatic Stress Studies, 2, 199-223. https: / / doi.org/10.1002/jts.2490020207

Shapiro, F. (1995). EMDR: Basic principles, protocols, and procedures. New York, NY: Guilford Press.

Shapiro, F. (2001). Eye movement desensitization and reprocessing: Basic principles, protocols and procedures (2nd ed.). New York, NY: Guilford Press.

Soberman, G., Greenwald, R., \& Rule, D. (2002). A controlled study of eye movement desensitization and reprocessing (EMDR) for boys with conduct problem. Journal of Aggression, Maltreatment, and Trauma, 6, 217236. https: / / doi.org/10.1300/J146v06n01_11

Strauss, A., \& Corbin, J. (1990). Basics of qualitative research: Grounded theory procedures and techniques. Newbury Park, CA: Sage.

Strauss, A., \& Corbin, J. (1994). Grounded theory methodology: An overview. In N. K. Denzin \& Y. S. Lincoln (Eds.), Handbook of qualitative research (pp. 273-285). Thousand Oaks, CA: Sage.

Taylor, S. J., \& Bogdan, R. (1984). Introduction to qualitative research methods (2nd ed.). New York, NY: Wiley.

Terr, L. (1991). Childhood traumas: An outline and overview. American Journal of Psychiatry, 148, 10-20. https: / / doi.org/10.1176/ajp.148.1.10

Van der Kolk, B. A. (1996). Trauma and memory. In B. A. van der Kolk, A. C. McFarlane, \& L. Weisaeth (Eds.), Traumatic stress: The effects of overwhelming experience on mind, body and society (pp. 279-302). New York, NY: Guilford Press.
Walker, J. L., Carey, P. D., Mohr, N., Stein, D. J., \& Seedat, S. (2004). Gender differences in the prevalence of childhood sexual abuse and in the development of pediatric PTSD. Archives of Women's Mental Health, 7, 111-121. https: / / doi.org/10.1007/s00737-003-0039-z

Ward, T. (2000). Sexual offenders' cognitive distortions as implicit theories. Aggression and Violent Behavior: A Review Journal, 5, 491-507. https://doi.org/ 10.1016/S1359-1789(98)00036-6

Ward, T., \& Hudson, S. M. (2000). Self-regulation model of relapse prevention. In D. R. Laws, S. M. Hudson, \& T. Ward (Eds.), Remaking relapse prevention with sex offenders: A sourcebook (pp. 39-55). Thousand Oaks, CA: Sage.

Ward, T., Hudson, S. M., \& Keenan, T. (1998). A selfregulation model of the sexual offense process. Sexual Abuse: A Journal of Research and Treatment, 10, 141-157. https: / / doi.org/10.1177/107906329801000206

Ward, T., \& Siegert, R. J. (2002). Toward a comprehensive theory of child sexual abuse: A theory knitting perspective. Psychology, Crime, and Law, 8, 319-351. https: / / doi.org/10.1080/10683160208401823

Warren, R. C., \& Green, M. T. (1995). Challenging the current paradigm amidst a culture of denial: Transformative treatment of sex offenders. Canadian Journal of Human Sexuality, 4, 299-309.

Wolpe, J. (1982). The practice of behavior therapy. New York, NY: Pergamon.

Disclosure. The authors have no relevant financial interest or affiliations with any commercial interests related to the subjects discussed within this article.

Funding. The author(s) received no specific grant or financial support for the research, authorship, and/or publication of this article.

Merci d'adresser toute correspondance concernant le présent article à Ronald J. Ricci, 69 Shelly Street, Erial, NJ 08081. E-mail: rricci@vt.edu 


\section{Annexe}

\section{Guide d'entretien post-traitement}

Les questions suivantes ont été utilisées pour guider un entretien semi-structuré, mené par un interviewer indépendant, avec le participant au groupe. Les questions de l'entretien sont conçues pour être en rapport avec l'objectif des questions de l'étude. Les questions primaires peuvent être approfondies avec des questions improvisées. Les questions primaires sont les suivantes:

1. Quelle a été votre première réaction en entendant parler de l'EMDR ?

2. Qu'est-ce qui vous a poussé à essayer l'EMDR ?

3. Décrivez votre première expérience lorsque le clinicien a utilisé l'EMDR avec vous ?

4. Quels avantages, s'il y en a eu, avez-vous retirés de l'utilisation de l'EMDR?

5. Quels inconvénients, s'il y en a eu, décririez-vous dans l'utilisation de l'EMDR?

6. Le traitement EMDR a-t-il affecté vos sentiments ou vos pensées à l'égard d'autres personnes?

7. Depuis que vous vous êtes engagé dans un traitement EMDR, quels changements généraux, le cas échéant, pourriez-vous décrire à votre sujet lorsque vous considérez les sentiments ou les comportements que vous pourriez lier à votre propre histoire de traumatisme?
8. Si vous considérez le contrôle des pulsions comme difficile pour vous, l'EMDR a-t-elle changé votre capacité à gérer ces pulsions ? Si oui, comment ?

9. Rappelez-vous: vous n'avez pas besoin de m'expliquer ou de me donner des détails si ça vous met mal à l'aise. Beaucoup de délinquants ont des difficultés avec des excitations sexuelles qu'eux-mêmes ou les autres considèrent comme anormales. Avez-vous remarqué des changements dans vos pensées ou vos schémas sexuels depuis que vous avez commencé l'EMDR ?

10. Depuis que vous avez commencé le traitement EMDR, avez-vous remarqué des différences dans vos émotions, vos réactions, vos relations:

a avec les autres personnes de votre programme de traitement?

$\mathrm{b}$ avec le travail qu'on vous demande d'effectuer dans le cadre de votre programme de traitement?

c avec votre famille? 